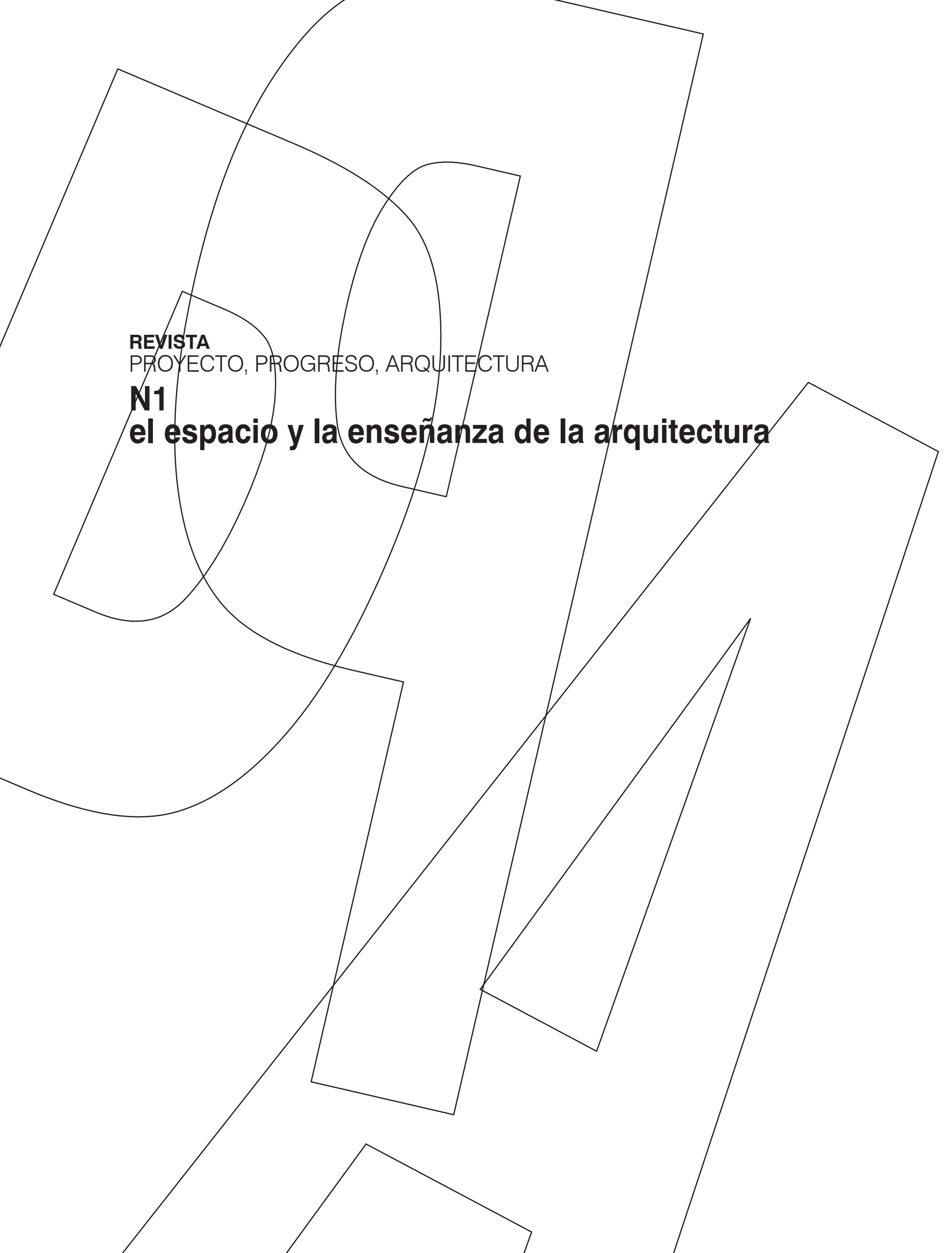


RESUMEN La propuesta docente surge de la necesidad de integrar las materias que intervienen en el proyecto de arquitectura y de la consideración de que un proyecto no es la suma de conocimientos desconectados e independientes. La Arquitectura es una actividad múltiple y compleja al tiempo que única y personal. Frente a la recepción pasiva de informaciones, la actividad arquitectónica como cualquier otra actividad creativa nos obliga a una verificación crítica de nuestro entorno y a la reivindicación de un conocimiento global síntesis de una identidad. El conocimiento, clasificado por tipos y materias, no puede nunca responder con éxito a la problemática amplia y diversa de una actividad que debe dar soluciones a un entorno complejo. La mejor docencia debería parecerse lo más posible a un estudio profesional de arquitectura en cuanto a la capacidad de integración de materias distintas. De aquí la condición global de la enseñanza que afecta a todo cuanto rodea al hombre, desde la tecnología hasta el clima y la cultura, los comportamientos sociales, la memoria o los sentidos.

PALABRAS CLAVE Docencia, coordinación, integración, simultaneidad, participación.

SUMMARY The course structure arises from the need to integrate the subjects involved in an architectural project and from the recognition that a project is not the sum of disconnected and independent knowledge. Architecture is a multiple and complex activity, whilst being unique and personal. As opposed to the passive reception of information, architectural activity, like any other creative activity, forces a critical verification of our surroundings and the recognition of a global knowledge synthesis of an identity. Knowledge alone, classified by types and subjects, can never successfully respond to the wide and diverse problems of an activity that must provide solutions to a complex environment. The best teaching should closely simulate a professional architectural studio regarding the capacity to integrate different subjects. This is the global condition of education that affects everything which surrounds humankind, from technology to climate and culture, social behaviours, the memory or the senses.

KEY WORDS Teaching, coordination, integration, simultaneousness, participation. 


\section{NUEVAS ILUSIONES PARA UNA ESCUELA}

NEW HOPES FOR A SCHOOL

Juan Domingo Santos odos los inicios son ilusionantes y los co$\mathrm{T}$ mienzos de la Escuela de Arquitectura de Granada en el año 93 estuvieron cargados de expectativas y de proyectos de futuro. A este prometedor arranque se sumó la convocatoria de un Concurso Internacional de Ideas para la creación de una futura escuela que fuera un referente para los estudiantes y para la ciudad de Granada. Transcurridos 15 años nos encontramos hoy ante la etapa más difícil de una escuela de arquitectura, el momento de su consolidación; y lo hacemos en unas condiciones precarias, sin un espacio adecuado donde desarrollar la docencia y sin unos medios básicos mínimos que aseguren la calidad de la enseñanza que se imparte. Lo que con ilusión comenzó a nacer y a tomar forma, hoy vive un estado crítico que debe hacernos recapacitar para tomar medidas de inmediato. Somos conscientes que la cantidad condiciona la calidad de una docencia, y nuestra escuela ha crecido en poco tiempo de forma desmesurada hasta alcanzar en la actualidad una cifra cercana a los dos mil estudiantes, un número por encima de lo recomendable para una escuela en una ciudad de reducido tamaño. En este proceso formativo y de consolidación hubiera sido deseable contar con un mayor apoyo por parte de las Administraciones competentes de la Junta de Andalucía y de la propia Universidad de Granada.

En la actualidad asistimos a dos modelos de escuelas de arquitectura. El modelo de macroescuela masificada dirigida por maestros que rara vez imparten clase y a los que difícilmente los estudiantes tienen acceso (es el caso del Politécnico de Milán o de Venecia, un modelo también adoptado en la escuela de arquitectura de Buenos Aires con tiendas, comercios y otros usos en el interior de sus instalaciones y un número de estudiantes superior a diez mil), y el modelo de escuela de reducido tamaño diseminadas por la geografía del país y con pocos estudiantes (entre trescientos y cuatrocientos), en las que el contacto directo del alumno con el profesor es su rasgo más característico (el caso de las escuelas alemanas y portuguesas). El modelo docente en nuestro país responde a una situación intermedia entre ambos, con un número de estudiantes que no debería sobrepasar nunca los mil doscientos, pero que desgraciadamente en la mayoría de los casos está muy por encima, con cifras que van de los dos mil hasta los cuatro mil estudiantes en escuelas como Madrid, Sevilla o Valencia, reguladas por un sistema de acceso con números clausus y una gran movilidad del estudiante en cursos superiores. El éxito de este tipo de escuelas radica en estar programadas convenientemente a fin de proteger su futuro, lo que significa proteger a su vez la calidad de la docencia, con un profesorado selectivamente elegido y una ratio nunca superior a treinta alumnos por profesor. En Granada hemos superado ya con creces las expectativas iniciales de crecimiento y lo más recomendable en este momento sería evitar saturarla, lo que daría lugar a una docencia masificada en pocos años. Ante esta situación el Consejo de Universidades debería tomar medidas drásticas que cualifiquen la formación futura del arquitecto. Si la demanda aumenta, en lugar de crecer y congestionar la actual escuela en detrimento de la calidad de su enseñanza, lo más acertado sería responder con la creación de una nueva escuela que nacería protegida y convenientemente programada. Somos conscientes de una demanda social que reclama calidad y una enseñanza más personalizada, aunque no existen suficientes recursos económicos ni de formación de profesorado. Pero la imparable masificación a la que tienden nuestras escuelas es un tema preocupante y su diversificación en el territorio una alternativa posible.

Sin embargo, y aún a pesar de esta difícil situación en la que nos encontramos, dos acontecimientos pueden devolvernos de nuevo la ilusión. Uno de ellos es la creación de un nuevo Plan de Estudios conforme al Plan de Bolonia, y el otro, la finalización de las obras para la futura Escuela de Arquitectura en un antiguo edificio situado en el Campo del Príncipe, un espacio histórico muy representativo de la ciudad situado a los pies de la Alhambra, con arquitecturas en su mayoría pertenecientes al siglo XIX e inicios del XX. Posiblemente Granada tenga en este momento la oportunidad única de llevar a cabo simultáneamente un Plan de Estudios y la construcción de una nueva Escuela de Arquitectura. La relación entre ambos es importante, ya que un plan de estudios no es ajeno al espacio arquitectónico y al lugar en el que se imparte, 
y Granada representa un lugar apropiado para vincular la escuela con la ciudad, su historia y sus habitantes. La calidad de una escuela radica en su especificidad y en lo que puede aportar frente a otras. En nuestro caso se trata de una propuesta implicada con la ciudad a través de la revitalización de su centro histórico. No hay mejor aprendizaje de la arquitectura que el propio edificio en el que se imparte, de aquí la importancia de llevar a cabo el proyecto de la futura escuela con las máximas exigencias arquitectónicas. Por otra parte ha sido también un acierto la decisión que tuvo en su momento el primer director de la escuela (Javier Gallego Roca) de ubicarla en la ciudad y no fuera de ella. En primer lugar porque el aprendizaje de la arquitectura pasa por una reflexión sobre la ciudad y nada mejor para el estudiante que vivir sus problemas cotidianos, y de otro lado por la capacidad revitalizadora y de implicación que supone para Granada la recuperación de un edificio de esta importancia con criterio y acierto. Una escuela de arquitectura en una ciudad pequeña como Granada debe estar arraigada con la ciudad y actuar como vínculo cultural con la sociedad. Una escuela comprometida de esta manera puede dar vida al entorno en el que se ubica y propiciar un campo de actividad que renueve un barrio histórico de la ciudad. El modelo de Campus Universitario fuera de la ciudad es un modelo en crisis, o al menos es una solución relegada a las grandes ciudades, pero no en nuestro caso.

El proyecto de nuestra futura escuela es una interesante apuesta de recuperación de un antiguo edificio y supone un potencial de extraordinario valor para la Universidad, para el barrio en el que se ubica y para la ciudad. Pensamos que se trata de un proyecto que puede devolvernos la ilusión al igual que el nuevo plan de estudios en el que durante más de un año hemos trabajado con intensidad profesores y alumnos a través de una comisión creada específicamente para este fin. Pretendemos que la escuela se convierta en un marco de relaciones múltiples, abierta a nuevas experiencias, y aspiramos a que sea un soporte cultural para la ciudad, un lugar donde mostrar y dar a conocer nuestras ideas y proyectos. En la actualidad de una escuela de arquitectura se busca que sea diferente, particular, que ofrezca una identidad reconocible que la haga distinta a otras. Nuestra intención es ocupar un antiguo edificio de mediados del siglo XVI con nuevas aportaciones desde la arquitectura contemporánea. Se trata de una construcción muy interesante que ha experimentado numerosas transformaciones a lo largo de la historia. En su origen un antiguo palacete (Casa del Almirante) ampliado en el siglo XVIII como Junta de Comercio, y más tarde Hospital de la Encarnación y Hospital Militar en el siglo XIX. El edificio contiene junto a algunos restos arqueológicos una estructura muy alterada por las sucesivas ampliaciones, destacando su capacidad para reordenar una trama urbana densa y compacta a través de la creación de nuevas edificaciones con patio. La intervención trasciende el propio edificio para vincularse a la ciudad. La apuesta es amplia y tiene también un alcance internacional por la manera de intervenir sobre lo patrimonial. La voluntad es que la actuación no se hiciera presente, que la mano del arquitecto no se hiciera notar. El proyecto ganador del concurso ha tenido varios parones prolongados en el tiempo y parece que por fin avanza en su recta final. El proyecto es obra del arquitecto Víctor López Cotelo y es un trabajo muy sensible que se hace desde el interior de la arquitectura como sucede en nuestra cultura. Se trata de la recuperación de un espacio en torno a dos patios, uno histórico muy bello con esbeltas arcadas y un salón principal con espléndido alfarje mudéjar, y otro patio más reciente pero de menor interés. Para explicar la intervención, Víctor López Cotelo habla de la luz, de la sombra, de los vacíos interiores, de limpiar añadidos hasta dejar su estructura muraria original. Habla de devolver el edificio a un estado en el que se puedan apreciar las huellas del tiempo, su memoria. Una intervención arqueológica y constructiva extendida a la arquitectura, descarnada en su estado más natural, donde convivirán estudiantes y profesores. El proyecto tiene una dimensión temporal y es muy sensible con el lugar y con su capacidad de transformación. Está formado por espacios abiertos comunicados entre sí que fomentan la participación integrada de estudiantes y profesores tal y como propone el nuevo plan de estudios. Este nuevo modelo de docencia abierto a nuevas experiencias requiere de un espacio libre y flexible donde poder llevar a cabo la 
enseñanza, como lo son los nuevos espacios de la futura escuela. Como novedad hay un espacio libre en primera planta, junto al vestíbulo de entrada y entre los dos patios, que se deja sin uso específico para desarrollar actividades múltiples de presentación de la propia escuela (trabajos PFC, propuestas y ejercicios de curso o exposiciones). Un espacio de comunicación interna y a la vez de comunicación con la sociedad, donde podamos dar a conocer las propuestas en las que trabajamos. El edificio acaba con el espacio clasificado y compartimentado como también propone el nuevo modelo de plan de estudios, abierto y participativo a la relación entre materias. Pretendemos que nuestra propuesta de plan tenga una perspectiva de 15 a 20 años en el marco de una escuela que acompañe este modelo docente, con aulas compartidas y fácilmente transformables según la actividad que se lleve a cabo en cada caso.

Respecto al nuevo plan de estudios, la reducción de créditos y horas lectivas implica un nuevo tipo de enseñanza que permita mantener los contenidos teóricos y prácticos de la carrera actual de arquitecto (de 400 créditos LOU en el Plan de Estudios actual se pasa a 300 ECTS para la obtención de grado más los 60 ECTS de Master habilitante para la profesión en los que se incluye el Proyecto Fin de Carrera como trabajo fin de master). Hemos pretendido que el nuevo Plan de Estudios para la Escuela de Arquitectura de Granada sea una propuesta avanzada que implique a profesores y estudiantes en un mismo proyecto por igual, y tiene como objetivo el aprendizaje de la arquitectura de una manera integrada entre las distintas materias. No presenta una metodología cerrada sino que surge de la experiencia abierta y de la relación entre conocimientos. Se pierde la multidisciplinariedad propia de un conocimiento clasificado y enciclopédico para pasar a un conocimiento que surge de la integración y de la experiencia de saberes. La tan recurrida "transversalidad" como conexión diagonal que atraviesa todos los estratos del conocimiento, se sustituye por un proceso de integración donde cada estrato, cada materia, deberá abrirse al conocimiento global. La propuesta docente nace de la consideración de que la arquitectura no es la suma de conocimientos desconectados e independientes. Pensamos que la mejor docencia debería parecerse lo más posible a un estudio profesional en cuanto a la capacidad de integración de materias distintas. El Plan se basa por tanto en una formación generalista del arquitecto, dejando para los másteres de postgrado la formación por especialidades.

Somos conscientes de que la formación técnica ha caracterizado al arquitecto español frente a otras titulaciones de arquitectura en Europa, rasgo que se mantiene en el nuevo Plan de Estudios como base de un proceso creativo fundamentado técnicamente. La visión conjunta y amplia del hecho arquitectónico nos ha diferenciado de otras enseñanzas y ha producido excelentes resultados en nuestra profesión. La conveniencia de interrelacionar conocimientos puede conducir a la creación de nuevas asignaturas en un intento por sintetizar una formación académica, sin embargo no parece necesario tener que inventar otras nuevas (aula-taller o laboratorio), ni cambiar la terminología actual para alcanzar una docencia completa, creemos que se puede lograr mediante la integración de las propias asignaturas que lo componen. En la actualidad el estudiante es el único que conoce la problemática de una enseñanza fragmentada que le hace trabajar en muchos casos repitiendo conocimientos ya adquiridos, o por el contrario, dejando vacíos inexplicables. El Plan se halla más próximo a la práctica pedagógica de formación "Bauhausiana", con una implicación interdisciplinar amplia y abierta que permite alimentar convicciones personales e influencias externas. La AA de Londres plantea una solución parecida mediante un sistema de "Unit" que incluye sesiones críticas, debates, viajes, jury, exposiciones, entre las actividades más destacadas.

Para llevar a cabo esta integración proponemos establecer previamente una serie de acuerdos entre materias, de programas y contenidos. La intención es que cuanta mayor coordinación se alcance entre el profesorado, mayor calidad en la docencia obtendremos. Esta coordinación entre materias se establecerá por semestres y por cursos, y deberá llevarse a cabo siempre a priori, nunca a posteriori ni durante el curso. Si la base de la organización docente es el semestre, la coordinación se realizará en torno a cinco asignaturas como máximo. Las reuniones de coordinación entre el profesorado servirán 
para el cruce de información, intercambio de opiniones y alcanzar unos objetivos comunes de programación. La información sobre los contenidos impartidos en materias como construcción o estructuras, por ejemplo, puede orientar el planteamiento del ejercicio de proyectos, y de la misma manera, desde la materia de proyectos se pueden formular sugerencias al resto de las materias.

La dificultad de la docencia ha sido siempre hacer simultáneos los aprendizajes integrados del conocimiento, cuando en el oficio de la profesión esta integración es uno de sus objetivos principales. La incorporación de conceptos como lo simultáneo frente a lo secuencial, es uno de los objetivos innovadores de esta propuesta de Plan de Estudios. La crítica a lo secuencial proviene de la visión codificada que se hace de la realidad reducida a una mera clasificación, ya sea por programas, escalas, tamaños o tipos, cuando la arquitectura es un proceso que atiende a un instante único de relaciones. La idea de lo simultáneo en la arquitectura hace posible una lectura más completa de la realidad, de aquí por ejemplo que los descriptores de las distintas unidades docentes en el Plan se presenten como espacios donde reflexionar y proponer soluciones, y no son más que un marco de trabajo para abordar la arquitectura de manera plena. Se podría describir la arquitectura como un acto único y complejo que aúna intereses de diferente tipo. Una acción simultánea de conocimientos que adquieren una forma precisa mediante el proyecto. Lo simultáneo aparece en el Plan vinculado a los temas de fondo de los descriptores, más libres y abiertos que las habituales clasificaciones docentes y a la metodología con la que se aborda la docencia, basada en la coordinación de materias por parte del profesorado y la integración de las mismas por el estudiante. Al habitual proceso de aprendizaje progresivo y clasificado por materias, escalas y conocimientos sucesivos, se opone este aprendizaje simultáneo en tiempo y espacio que implica una metodología docente más compleja, abierta y compartida. El proceso simultáneo de aprendizaje obliga también a un marco físico común de escuela, un espacio colectivo donde se abran a la experiencia las docencias con franjas horarias comunes y programas compartidos. La arquitectura trata de las relaciones entre cosas y sin esta relación el conocimiento parcial o fragmentado se limita a un campo muy reducido. Si avanzamos sólo en un conocimiento endógeno (la especialización) sin atender a la corrección que impone la síntesis del resto de componentes nos alejaremos cada vez más del tipo de aprendizaje generalista que pretendemos alcanzar para la profesión de arquitecto. Se olvida que cuando más se ensimisma el conocimiento en la especialidad, más nos alejamos de la visión global. El problema del especialista no es lo que conoce sino lo que olvida, lo que desconoce más que lo que sabe. El nuevo Plan pretende enseñar por tanto cosas parciales pero siempre vistas de manera conjunta, a través de una docencia activa, coordinada y simultánea.

Como novedad en este Plan, se incorpora en la formación básica del estudiante que se inicia en los estudios de arquitectura la asignatura "Ideación gráfica e introducción al proyecto arquitectónico", impartida conjuntamente por las docencias de Dibujo y de Proyectos, tradicionalmente muy vinculadas entre sí en la formación del estudiante y en la práctica profesional del arquitecto. La capacidad para representar y expresar gráficamente con el dibujo argumentos e ideas en el espacio constituye un aprendizaje fundamental para abordar el proceso de la creación arquitectónica.

Otra de las novedades de este Plan es la de partir de un programa horizontal conjunto confeccionado por todas las asignaturas que componen el semestre, compatible con los descriptores y ordenación de las materias implicadas. Un programa similar a las reuniones pluridisciplinares que se producen en la profesión ante cualquier problema real, desde el diseño de un objeto doméstico hasta la ejecución de un edificio con un programa complejo. La coordinación horizontal de la Unidad Docente puede completarse con una coordinación vertical que fomente la construcción curricular del estudiante, similar al sistema de las antiguas cátedras verticales. La flexibilidad de este modelo docente permite al estudiante decidir con libertad sus orientaciones futuras a la vez que fomenta una estructura docente horizontal compartida por el grupo.

El modelo docente que propone este Plan compromete a profesores y estudiantes a través de Unidades 
Didácticas, que deben suponer una mejora del aprendizaje. La unidad de trabajo será el semestre académico (dos por curso) en torno al cual se fomentará una enseñanza organizada que favorezca la relación entre los estudiantes. Cada semestre tendrá asociado un descriptor de trabajo y una Unidad Docente formada por diferentes grupos como base de la organización didáctica. Los grupos deberán establecer un programa y una metodología de trabajo teniendo en cuenta que el objetivo final es la integración de los conocimientos a través de una docencia activa e intensa.

Aunque en algunos casos se requerirá de ejercicios independientes en cada asignatura, las prácticas de las distintas materias de cada Unidad Docente se concentrarán en torno a la actividad proyectual del estudiante, ya que esta actividad genera su inquietud por adquirir los conocimientos necesarios. El conocimiento integrado por materias en el nuevo Plan es similar al tradicionalmente empleado en el Proyecto Fin de Carrera (PFC). El Plan añade al objetivo principal de verificación de los conocimientos adquiridos, un contenido docente final al contemplar el desarrollo del PFC mediante "aulas" que permiten completar y culminar el aprendizaje. Esta doble función del PFC, como complemento y práctica formativa de integración de conocimientos sectoriales y, finalmente, como examen o verificación de las capacidades y aptitud para el ejercicio profesional, viene justificada por la progresiva reducción de los planes de estudios de arquitectura a lo largo de los últimos cincuenta años.

Los másteres quedan fuera de la formación del estudiante para el ejercicio de la profesión y son un complemento y especialización posterior a la formación de grado, de aquí que deban estar vinculados a los programas verticales del Plan de Estudios y, al menos, alguna de las materias, pueda formar parte de las asignaturas optativas incluidas en la titulación. Los másteres a desarrollar deben tener en cuenta los específicos campos de trabajo de los arquitectos formados en esta escuela, el contexto físico, social y cultural en el que se implanta y las áreas de conocimiento relacionadas con la arquitectura, tanto en su componente social, como humanística y tecnológica.

\section{DESCRIPTORES Y CONTENIDOS}

El acercamiento al aprendizaje de la arquitectura puede producirse de muchas y variadas formas, de aquí que la designación de los descriptores de cada curso haga referencia a cuestiones amplias que enlazan la arquitectura con otros temas, convertidos en materia de investigación y estudio. La intención con los descriptores es establecer un marco de referencia donde el aprendizaje de la arquitectura aparece relacionado a las cuestiones de siempre: la ciudad, el patrimonio, la casa como hábitat, la colectividad, el paisaje o la técnica, que se convierten en asuntos de fondo tratados recurrentemente desde diferentes perspectivas. Se ha huido de la docencia clasificada por escalas y dificultad de programas, conscientes de que la arquitectura es un proceso complejo que en la actualidad abarca situaciones híbridas relacionadas con cuestiones de escala y de contenido.

El Plan se estructura en torno a cinco cursos y diez Unidades Docentes (300 ECTS), cada una de ellas correspondiente a un semestre académico, más un año de docencia destinado a la realización de un master habilitante (60 ECTS) que contiene un Proyecto Fin de Carrera como trabajo fin de master. Los descriptores de estas unidades didácticas de trabajo sobre los que se propondrán los programas docentes son los siguientes:

Las atenciones de la Arquitectura (Unidad Docente 1) y Realidad y representación (UD 2)

Son módulos de bienvenida del primer curso para el estudiante que se inicia en los estudios de arquitecto. Se trata de situar a la persona que se siente atraída por el aprendizaje de la arquitectura frente al debate de la misma. Se presentan los fundamentos de la arquitectura procurando el posicionamiento del estudiante ante la realidad sobre la que actúa, ante la relación sujeto/objeto que es propia de la interpretación y previa a la modificación de dicha realidad.

Forma, construcción y lugar (UD 3)

Se inicia una etapa en la que se reflexiona acerca de la naturaleza de las ideas y cómo surgen en el proceso 
creativo de la arquitectura. Argumentos que provocan acciones desencadenantes de un acto creativo y encuentran en la forma la traducción material de un pensamiento. El lugar aparece como la realidad física, pero también cultural o histórica. Se atiende a la naturaleza de la arquitectura como un proceso asociado a cuestiones de contexto, espacio, forma, función y técnica. La arquitectura dota de forma a las ideas y en este sentido se hace descriptiva.

\section{El hombre y la condición de habitar (UD 4)}

Se trata de indagar acerca de los contenidos genéricos del concepto habitar como un pensamiento y una actividad arquitectónica amplia que procuran la construcción física del mundo privado del sujeto, y el entendimiento del hábitat como un hecho arquitectónico y patrimonial.

Sociedad y ciudad I y II (UD 5 y 6)

El marco complejo en el que se produce la ciudad, su transformación y crecimiento arquitectónico son objeto de estudio y ensayo. La capacidad técnica y formal de la arquitectura para abordar los procesos de producción urbana e interpretar con acierto la complejidad del espacio público y privado y sus relaciones, descubrir las cualidades de lo colectivo frente a lo individual. Paralelamente a la visión simplificada de la ciudad por tipologías arquitectónicas y la zonificación por usos, encontramos espacios híbridos de actividad producidos por la intersección de intereses más amplios. La ciudad como un campo de relaciones, de intercambios.

Tiempo, transformación y patrimonio (UD 7)

Nos ofrece un campo amplio de reflexión y trabajo en el que la creatividad no está limitada a determinados lugares urbanos. La arquitectura moderna y contemporánea no debe identificarse sólo con la periferia de nuestras ciudades. Toda intervención sobre la arquitectura del pasado, sobre el patrimonio arquitectónico, nos remite a una combinación de conocimientos específicos con capacidad creativa sobre lo existente. El encuentro entre la arquitectura contemporánea y la arquitectura del pasado suele tener lugar en el interior de la ciudad histórica y supone una confrontación de tiempos y espacio a través del cambio y la permanencia.
Territorio y paisaje (UD 8)

Es posible que la diferencia entre los términos paisaje y territorio se encuentre en la aportación del hombre, que el paisaje no sea otra cosa que un territorio visitado y transformado por una acción, la interpretación y comprensión de una naturaleza. La relación naturaleza/acción (la representación) es un flujo complementario en ambos sentidos que va del registro de las identidades del territorio hasta el mapa, y a la inversa, ideas, argumentos y acciones que son trasladados a la naturaleza y provocan un modo particular de ocupación y rentabilidad del suelo. El paisaje de nuestro tiempo no es un paisaje puro ni estanco, se ha convertido en un lugar contaminado por la incidencia de numerosos factores e intereses entre los que se encuentran parajes residuales, industrias descontextualizadas o vacíos urbanos. La reflexión se centra sobre acciones y procesos que determinan una noción de paisaje independiente a la elaboración de formas y figuras a priori y en la que los procedimientos creativos de la arquitectura configuran una idea de paisaje no establecido.

Integración, sistemas y tecnología I y II (UD 9 y 10)

El compromiso con la dimensión técnica de la arquitectura adquiere un especial significado: de un lado alcanzar una postura crítica que podemos expresar en términos genéricos de razón e intención; y de otro, un entendimiento integral de la arquitectura y su aprendizaje como procesos inequívocamente orientados a la construcción de lo real.

Unidades docentes semestrales (UD)

Constituyen la base docente del aprendizaje integrado de la arquitectura. Las propuestas de trabajo de estas Unidad Didácticas deberá plantearse a partir de un Programa de Organización de la Unidad (POU), coordinado entre las materias que imparten la docencia en cada caso y en él se recogerán las aportaciones de cada materia así como todo tipo de actividades a desarrollar por el estudiante durante el semestre (sesiones teóricas y críticas, presentación de trabajos, viajes, conferencias, workshops, exposiciones...) Antes de iniciar el curso académico los grupos de cada Unidad Docente darán a conocer públicamente sus programas. Todos los POUs estarán 
incluidos en el Plan de Ordenación Docente (POD) de los diferentes departamentos y aprobados por la Junta de Escuela.

Los Programas de Organización de cada Unidad Docente enfatizarán procedimientos de enseñanza abiertos basados en la investigación y en la experimentación sobre los temas propuestos en los descriptores, y fomentarán la relación entre la teoría y la práctica. La Unidad Docente se convierte de este modo en un laboratorio de investigación abierto y un marco de acción donde debatir y proponer soluciones a los problemas cotidianos de la arquitectura. Conscientes de que la docencia no es una experiencia cerrada y que cada curso se presenta como la posibilidad para revisar, corregir y ajustar los conocimientos adquiridos, la Unidad Docente tendrá la posibilidad de renovar sus contenidos cada año a fin de mejorar su docencia en un proceso continuo de trabajo. Se deberá tender también a la estabilidad del profesorado que forme los grupos de cada Unidad Docente a fin de mejorar la calidad de la enseñanza.

El objetivo de la Unidad Docente es la producción de conocimiento, y al formar el estudiante parte de la misma, interviene en su formación, en la adquisición de la técnica y en la maduración del criterio arquitectónico necesario para abordar con cualidad la concepción de la arquitectura. Otros objetivos implícitos en la docencia son la estimulación de la creatividad personal y una cierta idea de compromiso colectivo. Desde esta doble perspectiva la pedagogía de la unidad docente tiene por finalidad facilitar el desarrollo de los aspectos subjetivos y creativos del estudiante, lo que determina una posición respecto a la disciplina y sus ámbitos de aplicación.

Juan Domingo Santos Granada (1961) Arquitecto por ETSA Sevilla (1986) Dr. Arquitecto por la Universidad de Granada (2005) y Profesor Titular en la ETSA de Granada. Ha impartido docencia en la Escuela Politécnica de Lausanne (Suiza, 1997), ETSA de Pamplona (2008), UIC de Barcelona (2009) y Technische Universität München (Alemania 2010). Su obra ha sido expuesta en la Bienal de Arquitectura Española (93-94); en la $7^{a}$ Mostra di Architettura Biennale di Venecia y en On-Site, New Architecture in Spain organizada por el MoMA de Nueva York. Su obra forma parte de la colección permanente de Diseño y Arquitectura del MoMA. Ha sido nominado a los Premios Mies van der Rohe 2007. 